\title{
Use of the Immunocrit to monitor a split-suckle program in commercial production
}

\author{
JL Vallet \\ USDA' ${ }^{1}$, ARS, U.S. Meat Animal Research Center (USMARC), Clay Center, Nebraska 68933, USA.
}

Neonatal piglets are born with limited energy stores and therefore require sufficient colostrum from the dam within the first $24 \mathrm{~h}$ of life to ensure their continued survival (Le Dividich et al., 1994). Many swine producers use split-suckling to optimize access of neonatal piglets to colostrum (Holyoake et al., 1995). However, it can be difficult to optimize the details of the procedures used and assess their outcomes with regard to colostrum acquisition by individual piglets. A technique called the "immunoglobulin immunocrit," based on measuring piglet serum immunoglobulin, was recently developed that allows rapid and inexpensive assessment of whether a piglet nurses successfully in the first day of life (Vallet et al., 2013). In collaboration with a commercial swine producer, the immunocrit was used to help assess the effectiveness of a split-suckle management program.

To assess the effect of split-suckling on the acquisition of colostrum by piglets, litters from sows (parity 1-8) were divided between control and split-suckle treatments in three separate replicates, providing data on a total of 151 control and 160 split-suckle litters (3,379 piglets). Split-suckle treatment occurred as follows: after a total of 9 piglets were born, the first 5 piglets born were separated from the sow by placing them into a heated box for up to $4 \mathrm{~h}$. This allowed the remainder of the litter better access to the sow. A visual assessment was made to ensure that the piglets removed from the sow had nursed successfully before removal. Control litters received no treatment. Blood samples were collected from piglets in each litter on day 1 of age. Blood samples were centrifuged and serum was measured for immunoglobulin using the immunocrit. Piglet weights were collected on day 1 and at weaning. Piglet mortality was recorded at weaning. In the second and third replicates, the fullness of the stomach of each piglet was also estimated by palpation at the time that blood samples were collected for the immunocrit $(0,50,100 \%)$, as a second method to determine whether piglets had successfully nursed.

The split-suckle treatment decreased $(P<0.05)$ the preweaning mortality of piglets born alive compared to untreated control litters (Table 1). Regression analysis of the relationship between birth weight and immunocrit values indicated that split-suckle treatment preferentially increased immunocrit values in low birth weight piglets $(P<0.05)$. Similar to previously reported results, preweaning mortality was affected by both birth weight $(P<0.01)$ and immunocrit values $(P<0.01)$, indicating that low birth weight and low immunocrits contributed independently to the risk of preweaning mortality. Examination of the interrelationships between birth weight, immunocrit values, and pre-weaning mortality indicated that survival decreased dramatically below an immunocrit threshold of 0.05 (the immunocrit is a ratio, it has no units). These results are very similar to those previously reported for piglets at USMARC (Vallet et al., 2013). Further analyses were done using 0.05 as a low threshold for the immunocrit. Split-suckle treatment tended to reduce the percentage of piglets below the 0.05 threshold, particularly in low birth weight piglets (Table 1). Analysis of the effect of split-suckle treatment on survival, after fitting either immunocrit values or whether immunocrit was below threshold, indicated that the reduction in mortality from split-suckle treatment was reduced when these covariates were included, but the effect of split-suckle treatment remained significant. This result indicates that 
the immunocrit accounts for some of the differences in mortality between control and splitsuckle treated litters, but differences in mortality between treatments remained after adjusting for these values. Finally, the influence of categorization of piglets based on fullness of the stomach assessed by palpation on the percentage of piglets that had immunocrits that were below the 0.05 threshold was examined. There was a significant effect of stomach fullness category on the percentage of piglets that were below threshold, indicating that these assessments were capable of detecting differences with regard to piglet acquisition of colostrum. However, in the group assessed as $100 \%$ full, a small percentage of piglets with immunocrits below the 0.05 threshold were still present. This suggests that palpation for stomach fullness was not a completely reliable method for determining whether a piglet received colostrum.

Table 1: Differences in preweaning mortality, average immunocrit, and percent of piglets below 0.05 threshold for the immunocrit are presented. Where indicated, means are for those piglets weighing more or less than $1 \mathrm{~kg}$ at birth or for piglets whose stomachs were palpated to be 0,50, or $100 \%$ full.

\begin{tabular}{lcc}
\hline Variable & Control & Split-Suckle \\
\hline$\%$ Preweaning Mortalitya & $15.1 \pm 1.5$ & $11.7 \pm 1.2$ \\
Average immunocrit $<1 \mathrm{kgb}$ & $0.093 \pm 0.004$ & $0.105 \pm 0.003$ \\
Average immunocrit $>1 \mathrm{~kg}$ & $0.105 \pm 0.002$ & $0.107 \pm 0.003$ \\
$\%$ below 0.05 threshold $<1 \mathrm{kgC}$ & $12.1 \pm 3.7$ & $7.3 \pm 2.7$ \\
$\%$ below 0.05 threshold $>1 \mathrm{~kg}$ & $4.5 \pm 0.8$ & $3.3 \pm 0.6$ \\
$\%$ below 0.05 threshold, $0 \%$ stomach fulld & $35.1 \pm 6.3$ & $21.1 \pm 5.4$ \\
$\%$ below 0.05 threshold, $50 \%$ stomach full & $8.1 \pm 2.8$ & $11.1 \pm 2.1$ \\
\hline below 0.05 threshold, $100 \%$ stomach full & $2.7 \pm 0.6$ & $1.8 \pm 0.5$ \\
\hline
\end{tabular}

a Effect of split-suckle treatment, $\mathrm{P}=0.078$.

b Split-suckle treatment by weight class interaction, $\mathrm{P}<0.05$.

${ }^{c}$ Effect of weight class, $\mathrm{P}<0.01$.

${ }^{\mathrm{d}}$ Effect of fullness assessment, $\mathrm{P}<0.01$.

These results indicate that split-suckle treatment reduced piglet mortality and improved piglet access to colostrum from the sow and that this improved access to colostrum contributed to reduced mortality. However, the results also indicated that a portion of the reduced mortality from split-suckle treatment is independent of colostrum access. The immunocrit was useful in determining the effectiveness of assessment of piglets by palpation with regard to the acquisition of colostrum. In conclusion, the immunocrit is useful for testing the effectiveness of various aspects of colostrum management techniques.

\section{References}

Holyoake PK, Dial GD, Trigg T, King VL. 1995. Reducing pig mortality through supervision during the perinatal period. Journal of Animal Science 73:3543-3551.

Le Dividich J, Herpin P, Rosario-Ludovino RM. 1994. Utilization of colostral energy by the newborn pig. Journal of Animal Science 72:2082-2089.
Vallet JL, Miles JR, Rempel LA. 2013. A simple novel measure of passive transfer of maternal immunoglobulin is predictive of preweaning mortality in piglets. Veterinary Journal 195:91-97. 